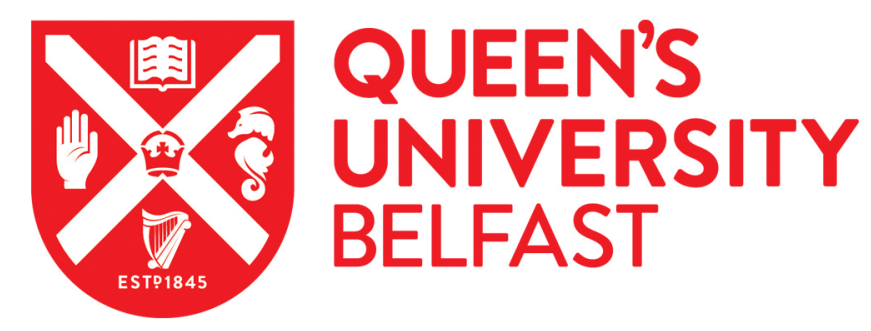

\title{
Sculptured Surface Oriented Machining Error Synthesis Modeling for five-axis Machine Tool Accuracy Design Optimization
}

Li, H., Li, Y., Mou, W., Hao, X., Li, Z., \& Jin, Y. (2016). Sculptured Surface Oriented Machining Error Synthesis Modeling for five-axis Machine Tool Accuracy Design Optimization. International Journal of Advanced Manufacturing Technology. https://doi.org/10.1007/s00170-016-9285-x

Published in:

International Journal of Advanced Manufacturing Technology

Document Version:

Peer reviewed version

Queen's University Belfast - Research Portal:

Link to publication record in Queen's University Belfast Research Portal

Publisher rights

Copyright Springer-Verlag London 2016

The final publication is available at Springer via http://link.springer.com/article/10.1007\%2Fs00170-016-9285-x

\section{General rights}

Copyright for the publications made accessible via the Queen's University Belfast Research Portal is retained by the author(s) and / or other copyright owners and it is a condition of accessing these publications that users recognise and abide by the legal requirements associated with these rights.

Take down policy

The Research Portal is Queen's institutional repository that provides access to Queen's research output. Every effort has been made to ensure that content in the Research Portal does not infringe any person's rights, or applicable UK laws. If you discover content in the Research Portal that you believe breaches copyright or violates any law, please contact openaccess@qub.ac.uk. 


\title{
Sculptured Surface Oriented Machining Error Synthesis Modeling for Five-axis Machine Tool Accuracy Design Optimization
}

\author{
Hai $\mathrm{Li}^{1}$, Yingguang $\mathrm{Li}^{1 *}$, Wenping Mou ${ }^{1}$, Xiaozhong $\mathrm{Hao}^{1}$, Zhixiang $\mathrm{Li}^{1}$, Yan Jin ${ }^{2}$ \\ (1 College of Mechanical and Electrical Engineering, Nanjing University of Aeronautics and Astronautics, \\ Nanjing, 210016, Jiangsu, China) \\ (2 School of Mechanical and Aerospace Engineering, Queen's University Belfast, Belfast, BT7 1NN, Northern \\ Ireland, UK)
}

\begin{abstract}
:
Customer-oriented design is very important for machine tool manufacturers to win competition in the market. Mechanical parts with complicated sculptured surface are widely utilized in mechanical systems such as automobiles, aircrafts and wind turbines, and they are often machined by five-axis machine tools with high precision requirements. However traditional machine tool design has not accounted for the varied machining errors in producing complex sculptured surface, which leads to inferior performance. To address this challenge, a novel machining error synthesis model is proposed in this paper for accuracy optimization in designing general five-axis machine tools used for making various sculptured surfaces. The new synthesis model bridges between surface machining profile error and the machine tool accuracy, and it is constructed by integrating a generic machine tool volumetric error model and two new surface machining error production models. The synthesis model is then applied as a constraint in machine tool accuracy design optimization. Also a tolerance-cost function is formulated to construct the objective function, and a meta-heuristic algorithm is developed to implement the optimization. All these modeling and optimization methods are validated by one case study.
\end{abstract}

Key Words: Accuracy design · Five-axis machine tool $\cdot$ Sculptured surface $\cdot$ Volumetric error $\cdot$ Tolerance

\footnotetext{
*Author to whom correspondence should be addressed.

E-mail: liyingguang@nuaa.edu.cn
} 
design

\section{Introduction}

Customer-oriented design of the machine tool is very important for machine tool manufacturers to win the competition in the market. Mechanical parts with complicated sculptured surface such as automobile moulds, aircraft components and turbine blades are often machined by five-axis machine tools, and their quality requirements are key sources for the customer-oriented design of tools.

Machine tool accuracy is one of the most important factors to be taken into account at the machine tool design phase, as the accuracy of a five-axis machine tool is one of the crucial factors affecting the design decisions on the quality of its individual parts and assemblies. Accuracy parameters of machine tool components are the basic measure for assessing the accuracy level of the five-axis machine tool, while they are specified in the international standard ISO230-6 or ASME B5.54. Furthermore, these desired accuracy parameters also restrain or guide the manufacturers' design and construction job of a machine tool directly. For example, from the aspect of the assembly process, these parameters are the assembly tolerances which specify the assembly requirements of the machine tool product. So, customer-oriented design is required in determining the design values of these dimensional and geometrical parameters in order to conform to customers' machining accuracy requirements.

In this paper, a sculptured surface oriented machining error synthesis model for five-axis machine tool accuracy design optimization is proposed. Section 2 reviews related literature. Accuracy parameters of five-axis machine tools are introduced in Section 3. The new synthesis model based on a general machine tool volumetric error model and two new surface error production models is proposed in Section 4, and it is then taken as the constraint condition for design optimization at the next section. In Section 5, tolerance-cost function is presented, as well as a search heuristic algorithm for machine tool accuracy design optimization. A case study is presented to validate the model and optimization method via simulations in Section 6 . Section 7 concludes the paper.

\section{Review of related work}


Machining error synthesis is a major way to construct optimization constraints for machine tool accuracy parameters design and optimization. Existing research in this area can be categorized into three classifications, i.e., Sensitivity Analysis [1,2], End Effector Accuracy Prediction [1,3] and Error Budget [1] of machine tools.

Sensitivity Analysis for machine tool errors is the study of how sensitive the output (machining sensitive directional error) of the machine tool can be resulted from different sources of uncertainty in its inputs (machine tool componement geometrical errors). Finding the sensitive machine tool errors, which are the most potential ones to improve the machining accuracy, is the main focus in this area. Chen [2] carried out the sensitivity analysis of volumetric error given 37 error components for designing a five-axis ultra-precision machine tool. Li [3] proposed an error modeling method based on multi-solid-body system theory to construct the error mapping between machine tool componement geometrical errors and the cutting tool pose error for a five-axis machine tool, and then the error sensitivity analysis of the five axis machine tool is conducted by the error mapping. The analyzed target of them is the plane surface or six directions of spatial coordinates, and the methods are not applicable for the sculptured surface because of uncertain sensitive directions.

End Effector Accuracy Prediction, as the name implies, is to predict the output accuracy of the machine tool end effector (such as the spindle or cutter tool) from the input of machine tool errors. Compared to the Sensitivity analysis field, the end effectors' accuracy prediction results (such as the roundness error of the spindle, or the cutter tools' position and orientation errors) are usually focused in this area to establish the error compensation job. Choi [4] predicted the roundness error of the spindle of a three axis machine tool through a volumetric error model. Okafor [5] constructed a kinematic model of a three axis machine tool to predict the cutter tools' position and orientation error for the error compensation, and Rahman [6] constructed kinematic model of a five axis machine tool for the error measurement. The cutter tools' position and orientation error could be calculated by these researches, and it is not enough for the final machining accuracy of the workpiece.

Error Budget can be defined simply as allocating allowable errors to meet a target machining accuracy 
[1]. Compared to the two methodologies above, the inner mechanism between the machine tool error input and machining accuracy output are more focused to improve machine tool accuracy level in the Error Budget area.

Error budget research is also reviewed by the authors from two aspects, which are the error budget considering the sensitive direction and the error budget considering feature and tolerance. There are plenty of the researches about error budget along the sensitive direction. Donaldson [7], Krulewich [8] and Walter [9] done the error budget along the axis the radial direction of the lathes machines to improve the lathing accuracy. Kroll [10] analyzed an X ray inspection machines mainly along the $\mathrm{X}$ axis. Erkorkmaz [11] analyzed a precision $\mathrm{X}-\mathrm{Y}$ working stage along the $\mathrm{X}, \mathrm{Y}$ and $\mathrm{Z}$ axis respectively, while Eisenbies [12] analyzed a CMM machine and Sun [13] analyzed an ultraprecision flycutting machine tool along the same axis direction. Error budget analysis on five-axis machine are done by Cheng [14], Brecher [15] and Treib [16] ,but their analysis are also along the $\mathrm{X}, \mathrm{Y}$ and $\mathrm{Z}$ axis respectively. Ibaraki [17] identified the machine tool errors by machining tests of stepped workpiece, and the map between the machine tool errors and workpiece errors is constructed. In summary, all above work is based on the sensitive direction, and they are not capable to deal with the machining accuracy synthesis of complex workpieces.

The error budget considering feature and tolerance are also reviewed by the authors, because the material remove modeling is also very important for the accurate finally parts' shape which is what the customers really wants. Feature is the way to describe the shape characteristics of the workpiece, and tolerance is the way to describe the nominal shape and position of the feature on the workpiece. Nominal tolerance of the feature is just the machining requirement when the machine tool is in machining. Some researchers study the error budget considering feature and tolerance. For example, Callaghan [18] proposed a machine error model to identify geometric errors from the prismatic feature tolerance for machine design.

The related research of the above three areas are summarized as shown in Table 1, which accounts for the general types of the mechanical machines, precision lathes, three-axis machines and five-axis machines.

Based on the comprehensive review, it can be concluded that the prismatic feature machining requirements oriented design optimization of five-axis machine tool accuracy parameters can be realized by the existed 
research $[17,18]$. Because the machining error synthesis modeling between the prismatic feature machining profile error (mainly the flatness error) can be naturally built by the error analysis along the normal direction (the sensitive direction) of a flat surface on a prismatic feature.

However, the five-axis machine tool errors' effect on sculptured surface profile machining errors can not be established by existing methods, because the above prismatic features' sensitive direction modeling is not valid. Therefore, the sculptured surface oriented machining error synthesis modeling for the five-axis machine tool accuracy design optimization is investigated in this paper, and the synthesis model is then used as the constraints in machine tool accuracy design optimization.

\section{Accuracy parameters of a five-axis machine tool}

From the aspect of the measurement, these accuracy parameters are mainly the quasi-static errors, which contains the geometric error and thermal errors, as reviewed by Ramesh [19]. What's more, from the aspect of the tolerance design and assembly process, these accuracy parameters are the assembly tolerances which specify the final assembly requirement of machine tool products. Here, the quasi-static error components, defined by multi rigidbody kinematics, are used to mathematically express these accuracy parameters. Later, these accuracy parameters will be the operands of the optimization problems in next section.

\subsection{Machine structure}

As shown in Fig. 1, A TTTRR(3 translational and 2 rotational DOF) type five-axis machine tool is taken as an example for the method of this paper. Five kinematic pairs including three translational axes and two rotational axes of the machine tool are the $\mathrm{X}, \mathrm{Y}, \mathrm{Z}, \mathrm{C}, \mathrm{A}$ axis respectively .The structure diagram and the kinematic chain diagram is according the definition in [20].

\subsection{Accuracy parameters}

The quasi-static errors of the machine tool stem from the errors of individual axes and those between axes..The quasi-static error components consist of positioning errors, straightness errors, angular errors, squareness errors and parallelism errors. As a rigid body has six degree of freedom, every translational axis or rotational axis has six quasi-static error components, which contain linear and angular errors. In a translational axis, the six error components are one linear positioning error, two straightness errors, and three 
angular errors called pitch, yaw, and roll respectively. In a rotational axis, the three linear error components are one axial error and two radial errors, and the three angular error components are one angular position error and two tilt errors. Also, there exists the squareness error between $\mathrm{X}$ and $\mathrm{Y}$ axis, $\mathrm{Y}$ and $\mathrm{Z}$ axis, $\mathrm{Z}$ and $\mathrm{X}$ axis, and the parallelism between $\mathrm{A}$ and $\mathrm{X}$ axis,and $\mathrm{C}$ and $\mathrm{Z}$ axis. Therefore, 37 quasi-static errors exist in the five-axis machine tool. These error components are listed in Table 2.

\section{Mapping model between machine accuracy parameters and machining accuracy}

The profile errors of the surface are caused by the errors in machining. Here, to restrain these machine tool accuracy parameters which are in form of quasi-static errors, the machine tool quasi-static errors' effect on the machined surface is investigated in this paper. Cutter's posture (include the position and orientation) errors, also known as volumetric errors [2], which are induced by the machine tool errors, will affect the profile errors of the machined surface. In other words, the cutter posture errors bridge between the machine tool errors and the profile errors of the machined surfaces.

\subsection{Volumetric error modeling of the machine tool}

Volumetric error modeling, which maps between machine tool quasi-static errors and cutter posture errors, is the first step in finding the relationship between machine tool errors and profile errors of machined surfaces. Rigid body kinematics and homogeneous transform(HTM) are used for volumetric error modeling in this section. Eight rigid bodies of a general five-axis machine tool shown in Fig.1 are defined. . Two structural chains exist in the machine tool. One is from the bed to the cutting tool via the machine tool, and the other is from the bed to the workpiece directly. These two chains are called "workpiece structural chain" and "cutting tool structural chain" respectively. Every rigid body on these two chains is coded by a number. The machine tool bed is usually associated with number zero. number 1 represents the first adjacent rigid body in the "workpiece structural chain", and the other numbers are used to represent the rigid bodies in the "cutting tool structural chain", as shown in Fig. 1. According to the rigid body kinematics in [2, 21], the transformation between the rigid body $k$ and its adjacent rigid body $j$ is described as 
where ${ }_{k}^{j} T_{p},{ }_{k}^{j} T_{p e},{ }_{k}^{j} T_{s}$, and ${ }_{k}^{j} T_{s e}$ are the posture transformation matrix, posture error transformation matrix, motion transformation matrix, and motion error transformation matrix between the rigid body $k$ and rigid body $j$, respectively. Based on Eq. (1), the transformation matrices between bodies in the workpiece structural chain and cutting tool structural chain can be obtained as follows.

$$
\begin{aligned}
& { }_{2}^{0} T=\left[\begin{array}{llll}
1 & & & P_{2 x} \\
& 1 & & P_{2 y} \\
& & 1 & P_{2 z} \\
& & & 1
\end{array}\right] I_{4 X 4}\left[\begin{array}{cccc}
1 & & & x \\
& 1 & & \\
& & 1 & \\
& & & \\
& & & 1
\end{array}\right]\left[\begin{array}{cccc}
1 & -\varepsilon_{\gamma}(x) & \varepsilon_{\beta}(x) & \varepsilon_{x}(x) \\
\varepsilon_{\gamma}(x) & 1 & -\varepsilon_{\alpha}(x) & \varepsilon_{y}(x) \\
-\varepsilon_{\beta}(x) & \varepsilon_{\alpha}(x) & 1 & \varepsilon_{z}(x) \\
& & & 1
\end{array}\right]
\end{aligned}
$$

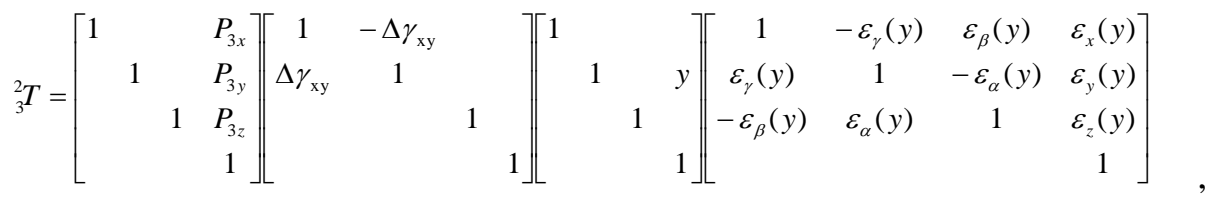

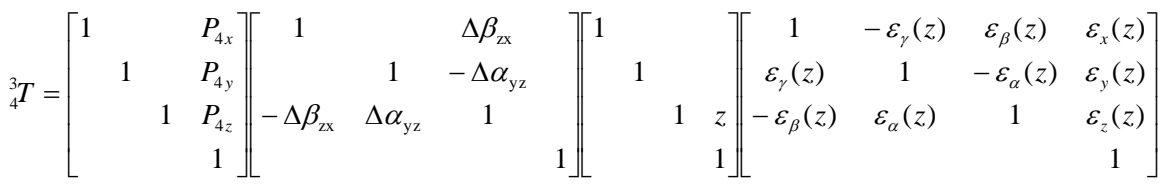

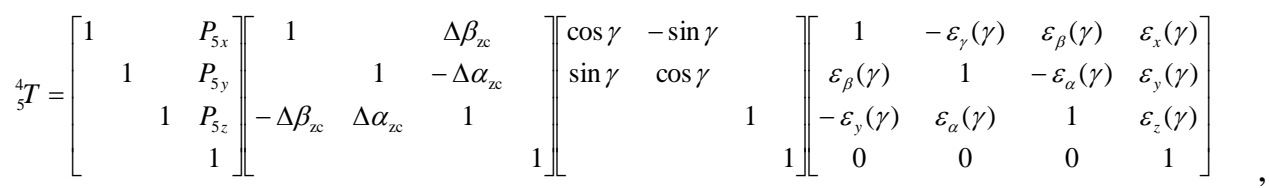

$$
\begin{aligned}
& { }_{6}^{5} T=\left[\begin{array}{cccc}
1 & & & P_{6 x} \\
& 1 & & P_{6 y} \\
& & 1 & P_{6 z} \\
& & & 1
\end{array}\right]\left[\begin{array}{ccccc}
1 & -\Delta \gamma_{x a} & & \\
\Delta \gamma_{x a} & 1 & \Delta \beta_{x a} & \\
-\Delta \beta_{x a} & & 1 & \\
& & & & 1
\end{array}\right]\left[\begin{array}{ccccc}
1 & 0 & 0 & 0 \\
0 & \cos \alpha & -\sin \alpha & 0 \\
0 & \sin \alpha & \cos \alpha & 0 \\
0 & 0 & 0 & 1
\end{array}\right]\left[\begin{array}{cccc}
1 & -\varepsilon_{\gamma}(\alpha) & \varepsilon_{\beta}(\alpha) & \varepsilon_{x}(\alpha) \\
\varepsilon_{\beta}(\alpha) & 1 & -\varepsilon_{\alpha}(\alpha) & \varepsilon_{y}(\alpha) \\
-\varepsilon_{y}(\alpha) & \varepsilon_{\alpha}(\alpha) & 1 & \varepsilon_{z}(\alpha) \\
& & & 1
\end{array}\right], \\
& { }_{7}^{6} T=\left[\begin{array}{cccc}
1 & & & P_{7 x} \\
& 1 & & P_{7 y} \\
& & 1 & P_{7 z} \\
& & & 1
\end{array}\right] \\
& { }_{1}^{0} T=\left[\begin{array}{cccc}
1 & & & P_{1 x} \\
& 1 & & P_{1 y} \\
& & 1 & P_{1 z} \\
& & & 1
\end{array}\right]
\end{aligned}
$$

where $P_{l x}, P_{l y}$, and $P_{l z}$ are the position coordinates of the workpiece's coordinate system relative to the bed's coordinate system (as shown in Fig. 2). $P_{i x}, P_{i y}$, and $P_{i z}(\mathrm{i}=2,3,4,5,6)$ in each transformation are the relative position coordinates of the ith body relative to the (i-1)th body. $P_{7 x}, P_{7 y}$, and $P_{7 z}$ are the relative position coordinates between the cutting tool and $\mathrm{C}$ axis. $x, y, z, \alpha, \gamma$ are the posture parameters associated with the 
five degree of freedoms. The rest accuracy parameters are shown in Table 2. The workpiece and cutting tool are fixed on the bed and the $\mathrm{C}$ axis, respectively, which is considered as no errors, as shown in Equation (7) and (8).

$\mathrm{R}_{\mathrm{w}}$ and $\mathrm{R}_{\mathrm{t}}$ are the position coordinate vectors of the cutting point in the coordinate systems of the workpiece and cutting tool respectively, which are expressed as

$$
\begin{aligned}
& R_{w}=\left[\begin{array}{lll}
R_{w x} & R_{w y} & R_{w z}
\end{array}\right]^{T}, \\
& R_{t}=\left[\begin{array}{lll}
R_{t x} & R_{t y} & R_{t z}
\end{array}\right]^{7} .
\end{aligned}
$$

$\mathrm{V}_{\mathrm{w}}$ and $\mathrm{V}_{\mathrm{t}}$ are the orientation vectors of the cutting point in the coordinate systems of the workpiece and cutting tool respectively, which are expressed as

$$
\begin{aligned}
& V_{w}=\left[\begin{array}{lll}
V_{w x} & V_{w y} & V_{w z}
\end{array}\right]^{T}, \\
& V_{t}=\left[\begin{array}{lll}
V_{t x} & V_{t y} & V_{t z}
\end{array}\right]^{T} .
\end{aligned}
$$

The cutter posture errors (include position and orientation) [Ex Ey Ez] and [Ei Ej Ek] are thenformulated as follows.

$$
\begin{aligned}
& {\left[\begin{array}{llll}
E_{x} & E_{y} & E_{z} & 1
\end{array}\right]^{T}={ }_{7}^{0} T \bullet\left[\begin{array}{ll}
R_{t} & 1
\end{array}\right]^{T}-{ }_{1}^{0} T \bullet\left[\begin{array}{ll}
R_{w} & 1
\end{array}\right]^{T}} \\
& {\left[\begin{array}{llll}
E_{i} & E_{j} & E_{k} & 1
\end{array}\right]^{T}={ }_{7}^{0} T \bullet\left[\begin{array}{ll}
V_{t} & 1
\end{array}\right]^{T}-{ }_{1}^{0} T \bullet\left[\begin{array}{ll}
V_{w} & 1
\end{array}\right]^{T}}
\end{aligned}
$$

where

$$
{ }_{7}^{0} T={ }_{2}^{0} T *{ }_{3}^{*} T *{ }_{4}^{*} T *{ }_{5}^{*} T *{ }_{6}^{*} T *{ }_{7}^{6} T \text {. }
$$

Therefore, equations (13) and (14) represent the mathematical relationship between the machine tool accuracy parameters and the cutter's posture errors based on the worst case scenario [reference required].

\subsection{Machining profile error modeling}

This section is to construct the analytical model between the cutter's posture errors and profile errors of machined sculptured surface.

\subsubsection{Profile error model of sculptured surface by end milling}

End milling is an important method for sculptured surface machining. In this subsection, the mathematical relationship between profile errors of the surface and the cutter posture errors will be constructed when in 
end milling. As the normal deviation, which is along the normal vector at the sample point on a sculptured surface, is usually utilized in measuring mechanical parts by a CMM(coordinate measuring machine), and then it could fully represent profile accuracy requirements of the surface [22]. If the normal deviation at every sample point is no more than the customers' permissible value, it's affirmed that the inspected surface satisfied the quality requirements. The mathematical relationship between these surface errors (the normal deviation) and the cutter posture errors will be constructed below.

Figure 3 shows that how the surface normal errors are affected by cutter tool posture errors at a sample point. As shown in Fig.3(1), if there are no cutter posture errors, the cutter contact point will be the sample point on the ideal surface. Because of the machine tool errors, the cutter center point will be away from its ideal position, and then there is an error space of the cutter center point caused by the cutter posture errors, shown as Fig.3(2). So, for the cutter posture errors, surface normal error and surface tangential error are generated as shown in Fig.3(3).

For describing the relationship between the cutter posture error and surface errors (normal deviation), as shown in Fig.4(1), $S_{0}$ is defined as the nominal surface, and $S_{1}$ is the actual surface after the machining within the cutter posture error. $P_{0}$ is the sample point on $S_{0}$, and the actual machining points corresponding to $P_{0}$ is the point $P_{l}$ on surface $S_{l}$. The deviation between $P_{l}$ and $P_{0}$ is the error $c$, which is the length of the cutter position error $[E x, E y, E z]$. Point $P_{2}$ on the normal direction line is the projection of $P_{1}$, then the projected length of error $c$ along the normal direction is assumed as $a$, the projected length along the tangential direction is assumed as $b$. The normal of $S_{0}$ at point $P_{0}$ intersect $S_{1}$ at the point $P_{2}$. The curvature radius of the surface at the sample point $P_{0}$ is $\rho$. Because the error $b$ as well as the changes of the surface normal $b / \rho$ is very tiny, we could consider the curve $P_{0} P_{0}$, as an arc curve. Then extend line $P_{l} P_{0}$ and $P_{2} P_{0}$, they intersect at points $O$, and the line segment $O P_{0}$ and $O P_{0}$,are equal length which is $\rho$.

1) Firstly, the normal deviation at the $P_{0}$, when cutting at $P_{l}$ is investigated. Due to the triangle $O P_{l} P_{2}$ in Fig.4(2) is a right triangle. The deviation $d$ shown in Fig.4(2) can be calculated as follow.

$$
d=\sqrt{\left(O P_{0}+P_{0} P_{2}\right)^{2}+P_{1} P_{2}^{2}}-O P_{0^{\prime}}=\sqrt{(\rho+a)^{2}+b^{2}}-\rho
$$


The magnitude $\left(10^{-2} \sim 10^{-3} \mathrm{~mm}\right)$ of error $a$ and $b$ is much smaller than $\rho$ (magnitude: $10^{1} \sim 10^{2} \mathrm{~mm}$ ), then,

$$
d=\frac{2 * a+a^{2} / \rho+b^{2} / \rho}{\sqrt{(1+a / \rho)^{2}+b^{2} / \rho^{2}}+1} \approx \frac{2 * a}{1+1}=a
$$

So, when the cutter is at the $P_{1}$, the normal deviation of the surface $S_{0}$ at $P_{0^{\prime}}$ is $a$, which is the projection of error $c$.

2) Secondly, the normal deviation at the $P_{0}$ when cutting at the point of $P_{2}$, on $S_{1}$ is investigated. As shown in Fig. 4(3), suppose $P_{3}$ is a point on the nominal surface $S_{0}$, and the corresponding points of $P_{3}$ is just the point $P_{2}$, on surface $S_{1}$ So $c^{\prime}$ is the deviation between $P_{2}$, and $P_{3}, a^{\prime}$ and $b^{\prime}$ are the projected length of $c^{\prime}$ along the normal and tangential direction respectively. Same as the reasoning process of step 1), when the cutter is at $P_{2}$, the normal deviation of the surface $S_{0}$ at $P_{3}$ is $a^{\prime}$, which is the projection of error $c^{\prime}$.

3) The error $c$ and the error $c$ ' are the length of the cutter position errors at point $P_{0}$ and $P_{3}$. According to the calculation by the Eq. (13) of section 4.1, the directional and value difference between error $c$ and the error $c^{\prime}$ could be ignored. So the normal deviation at $P_{0}$ could be considered as error $a$, which is the projected length of error $c$ (the length of the cutter position errors $[E x, E y, E z]$ ) along the surface normal.

So the normal deviation at the sample point $P_{0}$ is formed as follows.

$$
N d_{i}=x_{i}\left(x_{e 1}, x_{e 2}, \ldots x_{e 37}\right) * P_{i}+y_{i}\left(x_{e 1}, x_{e 2}, \ldots x_{e 37}\right) * P_{j}+z_{i}\left(x_{e 1}, x_{e 2}, \ldots x_{e 37}\right) * P_{k} \quad(i=1,2 \ldots n)
$$

where, $\left[P_{i}, P_{j}, P_{k}\right]$ is the normal direction coordinates at the sample point, $x_{e l}, x_{e 2} \ldots x_{e 37}$ is the value of the machine tool accuracy parameters, $x_{i}\left(x_{e 1}, x_{e 2} \ldots x_{e 37}\right), y_{i}\left(x_{e l}, x_{e 2} \ldots x_{e 37}\right), z_{i}\left(x_{e 1}, x_{e 2} \ldots x_{e 37}\right)$ is the coordinate components of the cutter position error $[E x, E y, E z]$ at the $i$ th sample point.

\subsubsection{Profile error model of sculptured surface by flank milling}

Flank milling is another important method for sculptured surface machining especially for the ruled surface. The mathematical relationship between the surface errors (the normal deviation) and the cutter posture errors when in the flank milling will be constructed in this subsection.

The geometrical intersection element between the cutter tool and surface is not a point but a short line segment when in surface flank milling, shown as Fig.5(3). The posture errors at any points on the short line 
segment can be calculated by the Eq. (13) of section 4.1. The difference in these points' cutter position errors calculation is only the difference of the cutter tool position transform coordinates $\left[p_{7 x} p_{7 y} p_{7 z}\right]$.Assume the cutter tool position transform coordinates at different points of the line segment are $\left[\begin{array}{lll}p_{7 x} & p_{7 y} & p_{7 z}\end{array}\right]_{P i}$ $\left(i=1.2 .3 \ldots . .\right.$. . As the line segment are very tiny (magnitude: $10^{1} \mathrm{~mm}$ ) compared to the cutter tool position transform coordinates at different points $\left[p_{7 x} p_{7 y} p_{7 z}\right]_{P i}(i=1.2 .3 \ldots$.$) (magnitude: 10^{3} \mathrm{~mm}$ ), Then the difference between the posture errors of every point on the line segment could be ignored through the calculation of Eq. (13).Then, a cutter contact points on the line segment are chosen to be analyzed in the following content to represent all the points on the line segment.

Same as the description of the end milling of Fig. 4 in subsection 4.2.1, that the error along tangential direction could be ignored, and the normal deviation at $P_{0}$ in Fig.6 could also be considered as error $a$, which is the projection of error $c$. So the normal deviation at $P_{0}$ is formed as follows, the same as equation (17).

$$
N d_{i}=x_{i}\left(x_{e 1}, x_{e 2}, \ldots x_{e 37}\right) * P_{i}+y_{i}\left(x_{e 1}, x_{e 2}, \ldots x_{e 37}\right) * P_{j}+z_{i}\left(x_{e 1}, x_{e 2}, \ldots x_{e 37}\right) * P_{k} \quad(i=1,2 \ldots n)
$$

where $x_{e 1}, x_{e 2} \ldots x_{e 37}$ is the value of the machine tool accuracy parameters, $x_{i}\left(x_{e l}, x_{e 2} \ldots x_{e 37}\right), y_{i}\left(x_{e 1}, x_{e 2} \ldots x_{e 37}\right)$, $z_{i}\left(x_{e 1}, x_{e 2} \ldots x_{e 37}\right)$ are the coordinates components of cutter position error $[E x, E y, E z]$ at the $i$ th sample point.

\section{Machine tool accuracy parameter optimization.}

Optimization techniques are the important ways to determine the tolerance allocation in mechanical design process. After the error synthesis model between the machine tool errors and profile errors of sculptured surface is constructed in the above section, the optimization constraints could be confirmed by this model. Objective function by tolerance-cost will be constructed to support the optimization process in this section, and the solution method of the optimization is also given.

\subsection{Optimization objective construction by tolerance-cost function}

From the view of the mechanical product assemblies, machine tool accuracy parameters, which are described through the geometric tolerance, are the requirements of the assembly processing. Hence, machine tool accuracy parameter-cost relationship can be regarded as the machine tool assembly tolerance-cost relationship. To optimize these tolerances, tolerance-cost models in the tolerance research are adopted to 
construct the objective function for the optimization.

\subsubsection{Tolerance-cost function}

Tolerance-cost function is the common method to describe the relationship between the manufacturing cost and the tolerance of the mechanical product to the assisttolerance optimization. There are several types of models of the tolerance-cost relationship, such as exponential model, reciprocal squared model, and reciprocal powers model [23]. The machine tool accuracy parameters in this paper could be considered as the assembly tolerance. Furthermore, the assembly cost is considered to be affected mainly by the fixed equipment cost and labor cost. For the description in the assembly tolerance allocation research [24], assembly tolerance-cost relationship must follow the following conditions: (a)When the tolerance $x_{\mathrm{i}}=0$, the cost $C\left(x_{i}\right)=\infty$,(b) the cost $C\left(x_{i}\right)$ decrease with increasing of tolerance $x_{\mathrm{i}}$, so the reciprocal powers model function is chosen to construct the cost-machine tool accuracy parameter function as follows.

$$
C\left(x_{i}\right)=\left(a_{i}+\frac{b_{i}}{x_{i}^{e_{i}}}\right)
$$

where the $C\left(x_{i}\right)$ is the fuzzy cost of the $i$ th parameter, $x_{i}$ is the $i$ th parameter, $a_{i}, b_{i}, e_{i}$ are the coefficient of the fuzzy cost for the $i$ th parameter. Specially, $a_{i}$ is considered as the fixed equipment cost, $e_{i}$ is the power

coefficient. For the errors belonging to the same axis of the machine tool, as shown in the previous Table 2, the related assembly job of the errors is in one work step, which is the assembly work step of the related axis. So, the coefficient $a_{i}, b_{i}$ and $e_{i}$ are the same at this time, while they are probably different if the errors belonging to different axes of interconnected machine tool components for the accuracy parameters. Also, how to gain these coefficients are shown in the next section 6 .

\subsubsection{Weight of the machine tool error}

The optimization problem for the machine tool error in this paper is a fuzzy comprehensive evaluation decision problem. The weight of each machine tool error need be considered to reflect the error's importance and influence to the resultant fuzzy cost, and this is very important to ensure the validity of the optimization results. The weights coefficients of every error are confirmed according to the Analytic Hierarchy Process (AHP) methods shown in literature [25]. The detail flow is as follows. 
The machine tool errors are generated through the relative motion between the machine tool components of each kinematic pair. Suppose $m$ is the total number of the kinematic pairs. As the assembly time is a represent of the assembly cost, assembly time is taken to construct the weights coefficients. The fuzzy cost weight $w_{j}$ of each kinematic pair could be:

$$
w_{j}=\frac{T_{j}}{\sum_{k=1}^{n} T_{k}}, j \in\{1,2, . ., m\}
$$

where, the $T_{j}$ is assembly time of the $j$ th kinematic pair.

Then, the total fuzzy cost weight of each error is considered like the follows.

Suppose $N$ is the total number of the errors of the machine tool, and the $i$ th error is a error belonging to the $N$ errors of the machine tool. Also, $n_{j}$ is the number of the errors belonging to the $j$ th kinematic pair, the $i_{j}$ th error is the a error belonging to this $j$ th kinematic pair, the total number $n_{j}$ is as shown in Eq.(21). The total number $N$ is the sum of the $n_{j}$, as shown in Eq.(22), and. And, if the $i$ th error of the machine tool is the $i_{j}$ th error of the jth kinematic pair, there will be Eq.(23).

$$
\begin{aligned}
& n_{j}=\sum_{\mathrm{i}_{j}=1}^{n_{j}} i_{j} \\
& N=\sum_{\mathrm{j}=1}^{m} n_{j} \\
& i=\sum_{\mathrm{k}=1}^{j-1} n_{\mathrm{k}}+i_{j}
\end{aligned}
$$

Then, consider the different difficulty when adjust the errors of the certain kinematic pair in the assembly job, fuzzy cost weight $\alpha_{i j}$ of the errors in the jth each kinematic pair are given to represent this difference. So the total fuzzy weight of the $i$ th error of the machine tool is:

$$
w_{i}^{f}=w_{i_{\mathrm{j}}}^{f}=\frac{\alpha_{i_{j}}}{\sum_{k_{j}=1}^{n_{j}} \alpha_{k_{j}}} * w_{j}, j \in\{1,2, . ., m\}
$$

where the $i$ th errors of the machine tool is the $i_{j}$ th error of the $j$ th kinematic pair, as shown in Eq.(23). 
Incorporating with the error-cost function in section 5.1.1, the total error-cost objective function of the machine tool is as follows, and the minimum value of total cost $C(x)$ is the optimization objective in this paper.

$$
C(x)=\sum_{i=1}^{n} w_{i}^{f} C\left(x_{i}\right)=\sum_{i=1}^{n} w_{i}^{f}\left(a_{i}+\frac{b_{i}}{x_{i}^{e_{i}}}\right)
$$

\subsection{Optimization constraints}

According to the customer's requirement, the normal deviation between detection point of the machined surface and the nominal surface should be less than the tolerance $T$. So the following inequation, which are the accuracy parameters optimization constraints, are formed.

$$
\operatorname{abs}\left(N d_{i}\right)<T,(i=1,2 \ldots n)
$$

where $N d_{i}$ is the normal deviation at the $i$ th sample points on the surface, which could be calculated by Eq.(17) and Eq.(18). The constraint is in the absolute value, shown as the calculation operator abs. Meanwhile, due to some design standards or experience, there are some value ranges of the machine tool accuracy parameters, as follows:

$$
\left\{\begin{array}{c}
e_{1 a}<x_{e 1}<e_{1 b} \\
e_{2 a}<x_{e 2}<e_{2 b} \\
\cdots \\
e_{37 a}<x_{e 37}<e_{37 b}
\end{array}\right.
$$

where $\left(e_{i a}, e_{i b}\right) i=1,2 \ldots ., 37$ are lower bound and upper bound of every accuracy parameter $x_{e i}$.

\subsection{Solving the machine tool accuracy optimization by search heuristic method}

Considering the optimization constrains function in section 4 and the optimization objective function in section 5, the optimization problem of this paper is a nonlinear programming problem with nonlinear constrains. As a useful search heuristic, Genetic Algorithm is chosen for solving these multivariable optimization problems, as shown in the next section.

\section{Case study}


Two surfaces, which could be machined by end milling method and flank milling method respectively, are used to construct the total constraint of the machine tool accuracy parameter optimization in this section. Based on the methodology in this paper, the optimization result and discussion will be presented below. And then, simulation was done to verify the correctness of the optimized results of the machine tool accuracy parameters.

\subsection{Discussions of the optimization results}

Five key points on each surface, shown in Fig. 7, are selected to build the total optimization constraints as described in subsection 5.2, and the machining requirement (normal deviation requirement) is $\pm 0.05 \mathrm{~mm}$. The reciprocal powers function is chosen as the tolerance-cost functions. The cost tolerance function coefficients values of $a_{i}, b_{i}$, and $e_{i}$ are selected from the standard data in literature [26], in which these coefficients are different when the manufacturing process are different or the manufacturing process size are in different ranges. After consulting with the machine tool manufacturers, the slideway length of $\mathrm{X}, \mathrm{Y}, \mathrm{Z}, \mathrm{C}$ and $\mathrm{A}$ axis (The rolling circumference of the rational axis of are considered as the slideway length) are given as $3 \mathrm{~m}, 2 \mathrm{~m}$, $2 \mathrm{~m}, 0.5 \mathrm{~m}, 0.3 \mathrm{~m}$. And we also consider the lapping process of the machine tool slideway is the main job in machine tool assembly, then the slideway length of every axes are considered as the lapping process size. So, according the lapping processes cost-tolerance functions data and the process size, we determined the values of $a_{i}, b_{i}$, and $e_{i}$, as shown in Table 3.Here the value $a_{i}$ is assumed to be $0 \$$, because it does not affect the optimization calculation, while this value actually depends on the industry, since the setup cost, equipment cost, etc., varies from industry to industry [27]. A series of values of the assembly time $T_{j}$ as described in section 5.1.1 are given also by consulting, as shown in Table 3. The range of every machine tool accuracy parameter is determined based on the machine tool design practices. Considering the importance of every accuracy parameter to the machine component is equal, the weight $\alpha_{i}$ is here assumed as the value of 1 , as shown in Table 3.

The optimization results are shown in the last column of Table 3. These results are the machine tool accuracy requirements over the present travels of every axis, which could then be used to as a design reference of machine tool accuracy parameters. Fig. 8 shows the best penalty fitness value and the mean 
penalty fitness value during the optimization process. It can be observed that the results are an optimal solution for the penalty fitness value is decreasing while the optimization process is convergent.

\subsection{Simulation verification}

To check whether the above results meet the requirements of the preset tolerance in section 6.1 , a simulation of the practical machining within the machine tool errors on the two surfaces are conducted using the Matlab development. The simulation procedure is shown in Fig. 9. Firstly, the surfaces from the mechanical parts are exported from a CAD software (NX8.0) as the original data. The original models have already been shown in Fig.7. Secondly, generate the machining tool path by the CAM software for the original surface, and the tolerance of the toolpath is set as $0.005 \mathrm{~mm}$, whose order of magnitudes is less than the machining tool errors' order. Thirdly, according to the simulation manner of robots [28], the machine tool error could be combined to the tool path by kinematic method to simulate the practical machining. Then, the surfaces are reconstructed through toolpath and tool contact points. The two simulated surface constructed by the original tool paths and tool contact points, are shown as in Fig.10(1) and Fig.11(1); The two simulated surface caused by machine tool errors are shown as in Fig.10(2) and Fig.11(2). At last, from comparing the two kinds of simulated surface with the ideal surface respectively, we could get the normal deviation error of the two kinds of surfaces. And then we could gain the toolpath tolerance's effect on sculptured surfaces' machining error and check whether the above optimized accuracy parameters results meet the requirements of the preset required tolerance.

Analysis of the surface were conducted using the Matlab language to obtain the toolpath tolerance's effect and the machine tool errors' effect on the surfaces' machining error, and the analysis result are shown on the vertical colorbar in Fig.10 and Fig.11. As in Fig.10(1) and Fig.11(1). The maximum normal error is no more than $0.005 \mathrm{~mm}$, and it shows the tool path computation error could be ignored in this simulation; In Fig.10(2) and Fig.11(2), the maximum normal error is no more than $0.05 \mathrm{~mm}$ and it shown the optimization results satisfy the preset required tolerance.

\section{Conclusions}

In this paper, a sculptured surface oriented machining error synthesis model based on a generic machine 
tool volumetric error model and two new machining error models is proposed. The error synthesis model will enable design optimization for accuracy parameters accounting customers' machining accuracy demand. An objective function is constructed based on tolerance-cost function, and an optimization algorithm based Genetic Algorithm is developed for optimizing machine tool accuracy parameters. A case study is studied and simulation results show both the model and the optimization approach are valid.

\section{Acknowledgment}

This research was supported by National S\&T Major Project Research Grant under No.2014ZX04014031.

\section{References}

1. Dornfeld D, Lee DE (2008) Precision Manufacturing, Springer.

2. Chen GD, Liang YC, Sun YZ, Chen WQ, Wang B (2013) Volumetric error modeling and sensitivity analysis for designing a five-axis ultra-precision machine tool. Int $\mathbf{J}$ Adv Manuf Technol 68(9-12):2525-2534

3. Li J, Xie FG, Liu XJ (2016) Geometric error modeling and sensitivity analysis of a five-axis machine tool. Int J Adv Manuf Technol 82:2037-2051.

4. Choi JP, Lee S.J, Kwon HD (2003) Roundness Error Prediction with a Volumetric Error Model Including Spindle Error Motions of a Machine Tool. Int J Adv Manuf Technol 21(12):923-928.

5. Okafor AC, Ertekin YM (2000) Derivation of machine tool error models and error compensation procedure for three axes vertical machining center using rigid body kinematics. Int J Mach Tools Manuf 40(8):1199-1213.

6. Rahman M, Heikkala J, Lappalainen K (2000) Modeling, Measurement and error compensation of multi-axis machine tools. Part 1: theory. Int J Mach Tools Manuf 40(10): 1535-1546.

7. Donaldson RR (1980) 'Error budgets' in technology of machine tools. Berkeley: Lawrence Livermore National Laboratory, UCRL-52960-5.

8. Krulewich DA (1998) 'A spatial-frequency-domain approach to designing precision machine tools' in Thrust Area Report.Berkeley: Lawrence Livermore National Laboratory, UCRL-ID-129204.

9. Walter M, Norlund B, Koning R, Roblee J (2014) Error budget as a design tool for ultra-precision 
diamond turning machines. Technical report of Precitech Inc.

10. Kroll JJ (2008) 'Error budgeting and certification of dimensional metrology tools' in FY07 Engineering Research and Technology Report. Berkeley: Lawrence Livermore National Laboratory, LLNL-TR-401927.

11. Erkorkmaz K, Gorniak JM, Gordon DJ (2010) Precision machine tool X-Y stage utilizing a planar air bearing arrangement. CIRP Ann Manuf Technol 59 (1):425-428.

12. Eisenbies SK, Hocken RJ (2000) Error Budget by Constraints. ASPE Proceedings, October 22-27, Scottsdale, Arizona.

13. Cheng X, Huang YM, Zhou SJ, Liu JY, Yang XH (2012) Study on the generative design method and error budget of a novel desktop multi-axis laser machine for micro tool fabrications, Int J Adv Manuf Technol 60(5-8):545-552.

14. Brecher C, Utsch P, Klar R, Wenzel C (2010) Compact design for high precision machine tools. Int $\mathbf{J}$ Mach Tools Manuf 50(4):328-334.

15. Treib T, Matthias E (1987) Error budgeting-applied to the calculation and optimization of the volumetric error field of multi-axis system. CIRP Ann Manuf Technol 36(1):365-368.

16. Sun YZ, Chen WQ, Liang YC, An CH, Chen GD, Su H (2015) Dynamic error budget analysis of an ultraprecision flycutting machine tool. Int J Adv Manuf Technol 76:1215-1224.

17. Ibaraki S, Sawada M, Matsubara A, Matsushita T (2010) Machining tests to identify kinematic errors on five-axis machine tools. PRECIS ENG 34(3):387-398.

18. Callaghan R (2003) Method for establishing machine tool performance specifications from part tolerance requirements. Transactions on Engineering Sciences: Laser Metrology and Machine Performance VI 44:507-516.

19. Ramesh R, Mannan MA, Poo AN (2000) Error compensation in machine tools-a review Part I: geometric, cutting-force induced and fixture dependent errors. Int J Mach Tools Manuf 40(9):1235-1256.

20. Bohez ELJ (2002) Five-axis milling machine tool kinematic chain design and analysis. Int J Mach Tools Manuf 42(4):505-520. 
21. Tian WJ, Gao WG, Zhang DW, Huang T (2014) A general approach for error modeling of machine tools. Int J Mach Tools Manuf 4(79):17-23.

22. Helmel Engineering Products Inc (2011) Curved Surface Measurement with Vector Point. Technical Note, January 15.

23. Chase KW (1999). Minimum-Cost Tolerance Allocation. Research Report of ADCATS(Association for the Development of Computer-Aided Tolerancing Systems), No.99-5.

24. Lin CY, Huang WH, Jeng MC, Doong JL (1997) Study of an assembly tolerance allocation model based on Monte Carlo simulation. J MATER PROCESS TECH 70(1-3):9-16.

25. Ayag Z, Ozdemir RG (2006) A fuzzy AHP approach to evaluating machine tool alternatives. J Intell Manuf 17(2):179-190.

26. Chase KW (1999) Tolerance allocation methods for designers. Research Report of ADCATS(Association for the Development of Computer-Aided Tolerancing Systems), No 99-6.

27. Prabhaharan G, Asokan P, Ramesh P, Rajendran S (2004) Genetic-algorithm-based optimal tolerance allocation using a least-cost model. Int J Adv Manuf Technol 24(9):647-660.

28. Rout BK, Mittal RK (2006) Tolerance design of robot parameters using Taguchi method. MECH SYST SIGNAL PR 20(8):1832-1852. 